\title{
The Interaction between Antitumor Drugs and a Double-stranded Oligonucleotide Studied by Electrospray Ionization Mass Spectrometry
}

\author{
Valérie Gabelica, Edwin De Pauw \\ Mass Spectrometry Laboratory, Chemistry Institute B6c, University of Liege, Liege, Belgium. \\ Frédéric Rosu \\ CHIMAPHY, Chemistry Institute B6c, University of Liege, Liege, Belgium.
}




\section{Abstract}

Electrospray ionization mass spectrometry has been used to investigate the complex formation between a double-stranded oligonucleotide and various antitumor drugs belonging to two categories: intercalators (ethidium bromide, amsacrine and ascididemin) and minor groove binders (Hoechst 33258, netropsin, distamycin A, berenil and DAPI). The goal of this study is to determine whether the relative intensities in the mass spectra reflect the relative abundances of the species in the solution phase. The full scan MS spectra suggest non-specific binding for the intercalators and specific binding for the minor groove binders. The preferential stoichiometries adopted by each minor groove binder have been determined by studying the influence of the drug concentration on the spectra. We obtained 2:1 > 1:1 for distamycin, 1:1 > 2:1 for Hoechst 33258 and DAPI, and only the 1:1 complex for netropsin and berenil. These features reflect their known behavior in solution. The compared MS/MS spectra of the 1:1 complexes with Hoechst and netropsin, when correlated to published crystallographic data, suggest the possibility to infer some structural information. The relative binding affinities of the drug for the considered duplex have been deduced with two by two competition experiments, assuming that the relative intensities reflect the composition of the solution phase. The obtained affinity scale is the following: netropsin > distamycin A > DAPI $>$ Hoechst 33258 berenil. These examples show some of the potential uses of mass spectrometry as a useful tool for the characterization of specific drug binding to DNA, and possibly a rapid drug screening method requiring low amounts of materials. 


\section{Introduction}

Mass spectrometry measurements on large molecules have been widely exploited since the introduction of electrospray ionization (ESI) $)^{1,2}$ and matrix-assisted laser desorption/ionization (MALDI) ${ }^{3,4}$ for the investigation of their primary structure by interpretation of the fragmentation patterns. Electrospray ionization, which is a soft ionization method (the analyte can be given little internal energy during the transfer to the gas phase), has furthermore opened the door to investigation of higher-order structures of molecules of biochemical interest ${ }^{5-7}$. The wide potentialities of mass spectrometry have been pushed to good use in protein research, from primary to quaternary structure ${ }^{8-12}$.

Nucleic acids have been far less investigated than proteins to date, but the interest in this field keeps growing (see refs. 13, 14, and references therein). Mass spectrometry has been used for primary structure determinations of oligonucleotides ${ }^{15,16}$ and for detection of highmass DNA samples ${ }^{17}$. The first observations of noncovalent duplex structures date back to $1993^{18-21}$ and the specificity of their formation has been discussed in 1995 by Ding and Anderegg $^{22}$. In 1997, small model oligonucleotide duplexes have been investigated by Williams et al. by the BIRD method ${ }^{23}$ : they correlated the stability of the duplexes in the gas phase with the number of hydrogen bonds between the strands, thereby inferring that the solution structure is in that respect maintained in the gas phase. Noncovalent interactions of molecules with the genetic material have also been investigated by electrospray ionization mass spectrometry: observation of interaction between small drug molecules and single strands ${ }^{24}$, hairpin $\mathrm{DNA}^{25}$ or oligonucleotide duplex ${ }^{26-28}$, interaction between proteins and oligonucleotides ${ }^{29,30}$ have been reported.

The study described herein is in keeping with the general pattern of determining the experimental conditions and the nature of molecules for which the informations obtainable with mass spectrometry are useful to solve problems relative to the solution phase behavior. There are two ways to consider that problem. First, one should determine the conditions in which, when recording a full scan MS spectrum, the nature of the species detected and their relative intensities reflect those in solution. This would allow the determination of the stoechiometries of the complexes and the binding constants in solution. Second, the manipulation of the complexes in the gas phase by MS/MS experiments allows the determination of energetic parameters, such as binding energies, in the gas phase. This feature could be particularly important in the case of multiple associations, where different 
contributions are difficult to sort out by solution phase measurements. It is also a probe of the supramolecular interaction in the absence of solvent that would lead to a better understanding of the solvent effect.

Oligonucleotide duplexes and their complexes with small molecules have been chosen as a model system. The comparison was performed between full scan spectra and what is known to be present in solution. The studied ligands belong to two classes of drugs: minor groove binders and intercalators. The common characteristics of minor groove binders are a flat, crescent shape, at least one positive charge, and many donor or acceptor groups. These features allow them to fit tightly in the minor groove of the double helix of DNA ${ }^{31,32}$. The complementarity between the acceptor/donor groups of the floor of the minor groove and the drug is at the heart of sequence recognition by synthetic molecules ${ }^{33}$. The studied ligands (scheme 1) have a higher affinity for regions with at least four consecutive $\mathrm{A}$ or $\mathrm{T}$ bases. Intercalator molecules, however, have a flat aromatic part which is able to stack between the base pairs of the duplex. Their binding to DNA is non-specific, with at the least a preference for GC-rich regions ${ }^{34,35}$. All of these drugs have either a well-established or a potential antitumor activity that is partially related to their binding to DNA. A very special effort is made nowadays in drug design to improve the sequence specificity of minor groove binders, with the final goal of targeting specific genes ${ }^{33,36-38}$. Structural characterization of the complexes are generally made by crystallography ${ }^{39}, \mathrm{NMR}^{40}$ or electric linear dichroism ${ }^{41}$, which are quite labour-intensive methods. Their thermodynamic characterization is usually performed by UV/visible spectrophotometry, NMR or fluorescence titrations ${ }^{42,43}$, or more recently by microcalorimetric methods ${ }^{44,45}$. In this article we will show which kind of informations can be readily extracted from mass spectrometry measurements, and their utility as a tool for the characterization of specific drug binding to DNA. 


\section{Experimental}

\section{Materials}

Oligonucleotide single strands were chosen purposely non self-complementary and of significantly different masses to allow unambiguous interpretation of the spectra. Single strand d(GGGGATATGGGG) will be referred to as $\mathbf{G}$ (monoisotopic mass = 3804.67) and the complementary strand d(CCCCATATCCCC) will be called $\mathbf{C}$ (monoisotopic mass = 3484.62). The resulting duplex (which will be called GC) is an analog of the well-studied self-complementary Dickerson dodecamer d(CGCGAATTCGCG) ${ }_{2}{ }^{46-49}$. All oligonucleotides were purchased from Eurogentec (Sart-Tilman, Belgium).

Minor groove binders (see scheme 1 for the structures and the monoisotopic masses) include Hoechst 33258 (Acros, Geel, Belgium), netropsin, distamycin A (Serva, Heidelberg, Germany) and berenil (kindly provided by C. Bailly (INSERM, Lille, France). Intercalators (scheme 2) are ethidium bromide, amsacrine (Sigma, Bornem, Belgium) and ascididemin (provided by C. Bailly).

\section{Sample preparation}

Oligonucleotides were diluted with freshly prepared $\mathrm{NH}_{4} \mathrm{Ac}$ solution, mixed in equimolar proportions, annealed at $80^{\circ} \mathrm{C}$ and slowly cooled to room temperature to allow the formation of the duplex. Desalting was performed three times (or more when the necessity was revealed by the spectra) with Microcon ${ }^{\circledR}$ filters (Amicon, Beverly, MA, USA) with a 3000 Da cut-off. The resulting duplex stock solution was $100 \mu \mathrm{M}$ in $50 \mathrm{mM}$ or $100 \mathrm{mM}$ $\mathrm{NH}_{4} \mathrm{Ac}$ (following the mass spectrometer, see below).

The electrolyte ammonium acetate has been chosen in agreement with the published literature. To determine its suitable concentration, we proceeded to thermal denaturation of the duplex in various $\mathrm{NH}_{4} \mathrm{Ac}$ concentrations. The concentration $50 \mathrm{mM}$ has been chosen for it stabilizes the duplex. For a $2 \mu \mathrm{M}$ solution in duplex, Tm is higher, $48^{\circ} \mathrm{C}$, in a $50 \mathrm{mM} \mathrm{NH}_{4} \mathrm{Ac}$ as compared to a $10 \mathrm{mM}$ solution, $37^{\circ} \mathrm{C}$ (figure 3 ). Circular dichroism measurements (data not shown) proved that in such conditions, although different than usual $\mathrm{NaCl}$ solutions traditionally used to study nucleic acids, the double helix is in a regular B-DNA form. In 
addition, ammonium acetate concentrations above $10 \mathrm{mM}$ have been previously shown to reduce single strand's charge states ${ }^{50}$, an interesting property because the lower the charge state, the lower the coulombic repulsion between the strands, and thus the more stable the complex.

Minor groove binders stock solutions were prepared by diluting the weighted solid in a $\mathrm{NH}_{4}$ Ac solution of appropriate concentration. Intercalators were dissolved in methanol to a concentration of $3 \mathrm{mM}$ before further diluting by aqueous $\mathrm{NH}_{4} \mathrm{Ac}$.

\section{Thermal denaturation}

Thermal denaturations were performed on a UV spectrophotometer Lambda 5 (Perkin Elmer, Norwalk, CT, USA) interfaced with a PC XT for data acquisition. The quartz cells were heated by a Julabo F25 heating circulator (Kutztown, PA, USA).

\section{Mass spectrometry}

Experiments were carried through with two different instruments. A Finnigan LCQ (ThermoQuest, Bremen, Germany) was used for full scan MS experiments. A Q-TOF (Micromass, Manchester, UK) was accessible when a resolution above 1,000 was required (competition experiments with drugs differing by only some daltons). In MS/MS experiments, the control of the collision energy was found easier with a quadrupole-TOF as compared to the LCQ.

With the LCQ, duplex solutions in 20/80 (v/v) methanol/100 $\mathrm{mM} \mathrm{NH}_{4} \mathrm{Ac}$ electrolyte were electrosprayed in the negative mode. Methanol was necessary to obtain a good spray, and circular dichroism revealed that $20 \%$ methanol did not alter the B double helix structure. The electrospray source conditions were optimized to favor the observation of the fragile noncovalent species. This implies that the ions should have undergone just enough collisions in the high-pressure region of the source to be fully desolvated, keeping their internal energy low enough not to be destroyed. We found that, when applying a heated capillary temperature of $190^{\circ} \mathrm{C}$, the complexes were intact and fully desolvated, so that a capillary-skimmer voltage of only few volts was sufficient to transfer the ions in the lower pressure region. Although complexes survive to cone voltages up to $-50 \mathrm{~V}$, we used a constant value of $-6 \mathrm{~V}$ to have a 
higher signal to noise ratio. The scanned mass range $(1,000-2,000)$ was also kept constant in every experiment. For competition experiments, the considered species are in the range [1450-1550], so that no significant effect on the intensities due to the $q_{z}$ values is suspected. The absence of discrimination was checked by comparing LCQ and Q-TOF results.

With the Q-TOF, the Z-Spray source was used to allow the analysis of duplex solutions in $50 \mathrm{mM} \mathrm{NH}_{4} \mathrm{Ac}$ without addition of methanol. The experimental conditions were optimized for the Z-Spray (a totally different source geomerty) with the same principles in mind : a source block temperature of $80^{\circ} \mathrm{C}$ and a cone voltage of $50 \mathrm{~V}$ were used (higher charge states could be additionnaly obtained under softer conditions, but the incomplete desolvation resulted in a noisy spectra, see below).

The DNA concentration has been optimized to $10 \mu \mathrm{M}$. It was observed that the duplex/single strands ratio rises when diluting the sample. However, the signal is decreasing subsequently and the spectra become more noisy. An even more interesting feature of the dilution experiment is the partial suppression of sodium adducts when the duplex concentration is lower or equal to $10 \mu \mathrm{M}$ (see the insets of figure 1 ). This indicates that the $\mathrm{Na}^{+}$counterions are displaced by $\mathrm{NH}_{4}{ }^{+}$, and that a $\left[\mathrm{NH}_{4}{ }^{+}\right] /[$duplex] ratio of at least 5,000 is preferable. Ammonium adducts are readily eliminated by $\mathrm{NH}_{3}$ loss during desolvation, which simplifies the spectra. 


\section{Results and Discussion}

\section{Specificity of Duplex Formation}

The characteristics that allow to differentiate the specific associations observed by electrospray from non-specific ones have been discussed by Smith and Light-Wahl ${ }^{51}$ : these include persistence upon dilution, stability in the gas phase, and sensitivity to solution modifications. For double-stranded DNA, non-specific aggregation is reflected in random associations of non-complementary strands. These non-specific aggregations are often due to

concentration effects. Ding and Anderegg ${ }^{22}$ have demonstrated that they were avoidable by working at low concentration (around $10 \mu \mathrm{M}$ ) with reasonably long strands (octamers). This is confirmed by our study of the effect of the concentration : the non-specific homodimers $(\mathrm{GG})^{5-}$ and $(\mathrm{CC})^{5-}$ that were still present at very low intensity at $25 \mu \mathrm{M}$ concentration (fig. 1top) completely disappeared at $10 \mu \mathrm{M}$ concentration (fig. 1-center), while the relative intensity of the specific duplex increased compared to the single strands.

The gas phase stability of the duplex has been probed by MS/MS on different charge states (Fig. 2). A capillary-skimmer voltage of $30 \mathrm{~V}$ (instead of 50) was chosen to maintain intact the $(\mathrm{GC})^{6-}$ species for its selection in MS/MS. As expected, the collision energy necessary to fragment $50 \%$ of the duplex depends on the charge state. The adequate acceleration voltage applied before entering the quadrupolar collision cell varied from $19 \mathrm{~V}$ for $(\mathrm{GC})^{6-}$ to $25 \mathrm{~V}$ for $(\mathrm{GC})^{5-}$, in agreement with the higher stability of lower charge states. The discrepancy between the absolute values of the different voltages used is only apparent: the voltage on the sampling cone, on the contrary to the voltage used for CAD, cannot be translated in terms of kinetic energy of the ions. In fact, in the source, the voltage on the cone induces the drag of the ions through the atmospheric pressure gas where multiple collisions occurs at low energy. This activation process is referred to as slow heating ${ }^{52}$. The fragments resulting are the single strands, between which the available charges are shared. In addition, strand G has a greater tendency to leave protons to the other strand, therefore resulting in higher charge states than strand $C$. This is in agreement with the lower pKa value for guanine than cytosine ( 2.1 vs 4.2 ). The relatively high values of the collision energy necessary to fragment the duplex (single strands are already fragmenting to a significant extent at $30 \mathrm{~V}$, data not shown) are indicative of the specificity of the analyzed complex. Indeed, non-specific 
complexes resulting from random aggregation in the gas phase are typically weak, and are readily fragmented at low collision energies.

To show how closely the mass spectra can be an image of the species present in the sprayed solution, we compared a freshly annealed solution in $50 \mathrm{mM} \mathrm{NH}_{4} \mathrm{Ac}$ with a solution left overnight at $37^{\circ} \mathrm{C}$ in a $10 \mathrm{mM} \mathrm{NH}_{4} \mathrm{Ac}$ solution $\left(\mathrm{T}_{\mathrm{m}}=38^{\circ} \mathrm{C}\right)$, sprayed immediately without cooling. In this solution, there should be approximately half the amount of duplex than in the freshly annealed one (see fig. 3). The MS results (fig. 4) show that the relative intensity of the duplex compared to the single strands is indeed reduced by a factor of about two in the partially denatured sample. This observation strongly supports that the relative intensities of the noncovalent duplex peaks observed in the gas phase closely reflect the composition of the solution. The increase of sodium adducts in the latter spectrum indicates again that lower $\mathrm{NH}_{4}$ Ac concentrations (10 mM instead of $50 \mathrm{mM}$ ) do not efficiently displace the sodium from the negatively charged ions in solution as mentionned above.

\section{Complexes with Minor Groove Binders}

Stoichiometry - Once having set up the optimal conditions for the observation of the specific duplex, they were used for the study of the non-covalent interactions between the duplex and the minor groove binders displayed in scheme 1. Figure 5 shows the spectra obtained with the LCQ for the 1/1 drug-duplex mixtures. Different behaviors can be distinguished for the different drugs following their spectra. Berenil and netropsin show exclusively the 1:1 complex, DAPI and Hoechst 33258 show a small amount of 1:2 complex in addition to the 1:1 one, and for distamycin A the 1:2 complex is the predominant species.

To further characterize the formation of the 1:1 and 1:2 complexes, we studied the influence of the concentration of drug, from $2 \mu \mathrm{M}$ to $20 \mu \mathrm{M}$, added to the $10 \mu \mathrm{M}$ duplex solution in the case of netropsin, Hoechst 33258 and distamycin A. The data are summarized in figure 6 , where the relative intensities have been normalized to $100 \%$ for each spectra. For netropsin, no 1:2 complex could be observed, even at the highest drug concentrations. For distamycin A, the 1:2 complex becomes rapidly predominant as the drug concentration increases, thereby indicating the preferential one-step formation of this 1:2 complex over the 1:1 complex, whose signal vanishes progressively. The distinct behaviors of netropsin and distamycin A are well-known in the solution phase ${ }^{31}$. Distamycin forms a head-to-tail dimer which inserts in the AT-rich region, thereby widening the minor groove ${ }^{53-54}$. This 1:2 binding 
mode is favored by an alternating AT sequence compared to poly(A)-type ones because they naturally present a wider and more flexible minor groove ${ }^{31,55}$. In the case of netropsin, however, the positive charges present at each end of the molecule prevent the formation of such dimers and the resulting complex with the AT-rich region is thus exclusively 1:1.

In the case of Hoechst 33258, mass spectrometry is shedding a new light on a controversial problem: the possible existence of multiple stoichiometries for Hoechst binding to DNA ${ }^{56-58}$. With measurements in solution, in general, the presence of minor species of different stoichiometries is only suggested by clues. For example, a fluorescence measurement that cannot be fitted with a 1:1 model may be indicative that other complex species are present, but no clear cut can be made because the contributions of the different complexes to the total fluorescence can't be separated. Our results suggest the formation of a 1:2 complex with a lower binding constant than for the 1:1 complex, which is the predominant one. Mass spectrometry has the advantage of direct assignment of the stoichiometry, but it can also give an indication about the relative amounts of the different species. In this way of thinking, we are assuming that the relative intensities observed by ESIMS reflect the relative abundances in solution. This argument has been used in various studies $^{59-62}$ and is thought to be valuable when the observed complexes are likely to be stabilized in the gas phase compared to the solution phase, mainly by electrostatic and hydrogen bonding interactions ${ }^{63-64}$. This is indeed the case for the binding of minor groove ligands with double-stranded DNA.

Competition experiments - With the same way of reasoning, competition experiments were undertaken: the duplex was mixed with equal amounts of two drugs in a 10-10-10 $\mu \mathrm{M}$ solution, and the spectra of the mixtures were recorded. Some are reported in figure 7 . The competition experiments performed on the LCQ were made for drugs of significantly different masses. The competition between Hoechst 33258 and netropsin required the resolution of the Q-TOF. These competition experiments allow the easy determination of which drug among the two ones tested binds the duplex preferentially. For example, it is readily inferred from spectrum (b) that berenil has approximately the same binding affinity as Hoechst 33258, and from (a) that DAPI binds the duplex with higher affinity. We can therefore step by step establish a relative affinity scale of drugs for a given duplex. In our case, we obtained the following scale: netropsin > distamycin A > DAPI > Hoechst 33258 berenil. Thermal denaturation experiments showed that the complex with netropsin is indeed more stable than the one with Hoechst 33258 (fig. 8): the more stable the complex, the higher 
the denaturation temperature ${ }^{65}$. This possibility opens the door to many useful applications in the field of drug design, but some work is still needed to lead to quantitative measurements, with the support of microcalorimetric data. The improvement brought by a selected modification of a drug can be tested by mass spectrometry by a competition experiment between the modified molecule and the original one.

$\boldsymbol{M S} / \boldsymbol{M S}$ - To investigate the gas phase structure and stability of the (1:1) complexes, we performed MS/MS experiments on the (GC+drug) ${ }^{5-}$ species for Hoechst and netropsin (fig. 9). As the comparisons were made between the duplex and the different complexes only one charge state was monitored. The 5- charge state was chosen for MS/MS experiments, as allowing a suitable collision voltage range. The effect of the charge state on the MS/MS behavior is under investigation as it can probe the coulombic contribution of the interaction. The CID of the complexes in the 5- charge state shows a different behavior following the studied drug. The complex (GC+Hoechst) ${ }^{5-}$ dissociates at $30 \mathrm{eV}$ into the duplex and the single strands, with release of the neutral drug. The complex with netropsin $(\mathrm{GC}+\mathrm{Net})^{5-}$, however, dissociates at the same collision energy into the single strands with the netropsin drug remaining attached on strand $\mathrm{G}$. The magnitude of the collision energy needed to fragment the $(\mathrm{GC}+\mathrm{drug})^{5-}$ complexes reveals that they are more stable than the duplex alone. This parallels the feature, well-known in the solution phase, that minor groove binders stabilizes the double helix structure by hydrogen and Van der Waals bonding to both strands ${ }^{66}$.

Furthermore, could the different dissociation channel reflect structural features of the drug binding to this particular dodecamer? Part of the answer has been found in crystallographic structures of similar complexes. Netropsin and Hoechst are known to bind to the minor groove in the AT rich regions of the $\mathrm{DNA}^{31-33}$, and therefore in the ATAT region of our dodecamer. Crystallographic structures of the complexes between the self-complementary dodecamer d(CGCGATATCGCG) 2 and netropsin ${ }^{67}$ or Hoechst ${ }^{68}$ have been published and hydrogen bond number and lengths have been calculated (fig. 10). Hoechst forms three hydrogen bonds involving $\mathrm{NH}^{\cdots} \mathrm{O}$ interactions (whom one is tight : $2.9 \AA$ ) plus one weak $\mathrm{CH}_{2} \cdots \mathrm{O}$ interaction with the duplex. In contrast, netropsin has four $\mathrm{NH} \cdots \mathrm{O}$ or $\mathrm{NH} \cdots \mathrm{N}$ hydrogen bonds with the duplex, whom one is very tight (2.6 $\AA$ ). This simple count of the hydrogen bonds in the crystallographic structure of an analog dodecamer with the same binding region (ATAT) provides an explanation for the relative stabilities of the two complexes: complex with netropsin is more stable than complex with Hoechst because it forms more hydrogen bonds with the duplex. The difference in the fragmentation behavior of 
the two $(\mathrm{GC}+\mathrm{drug})^{5-}$ species can also be explained the same way: the balance of the number of hydrogen bonds with the two strands is more equilibrated in the case of Hoechst (2-1) than in the case of netropsin (3-1). Netropsin has therefore a greater tendency to remain attached to one of the strands when fragmentation occurs in the gas phase. These results show the potentiality, well worth to be further investigated, that fragmentation patterns upon CID could reflect structural features of the noncovalent bonding between molecules.

\section{Complexes with Intercalators}

Stoichiometry - The complexes between the three ligands displayed in scheme 2 and the duplex have been investigated by ESI-MS on the ion trap instrument. Figure 11 shows the spectra recorded for the three drugs at $10 \mu \mathrm{M}$ and $30 \mu \mathrm{M}$ concentration, the duplex concentration being $10 \mu \mathrm{M}$. The spectra reveal a different binding mode than minor groove binders: instead of well-defined stoichiometries, the intercalators show multiple associations with a distribution suggesting a non-specific behavior, which is compatible with their behavior in solution. The relative intensities complexes/duplex for the different drugs parallels their polarity: peaks from complexes with the positively charged ethidium are far more intense than those with the polar amsacrine, which are in turn more intense than those with the quasi non-polar ascididemin molecule. This could be an artifact due to the electrospray process itself. Either the complexes are formed during the electrospray process, and this complex formation is favored with more polar molecules when the dielectric constant diminishes ; or the complex is destructed in the gas phase, the less polar the ligand, the less stabilized the complex when the solvent contribution is absent. Solution binding constants will be required to drive our interpretation, but these results indicate that in the case of intercalators, the intensities of the peaks may not reflect the abundances in solution, but other characteristics of the drug, like its polarity.

Displacement of ethidium by minor groove binders - The concept of competition experiments has been applied to ethidium bromide: a duplex-ethidium $10 \mu \mathrm{M}-15 \mu \mathrm{M}$ mixture has been allowed to react with various amounts ( 0 to $15 \mu \mathrm{M})$ of minor groove binder at least 5 minutes (which turned out to be the minimum time to establish the equilibrium). Analog measurements are routinely achieved in the solution phase to determine the binding constant 
of the minor groove binder, provided that ethidium's one is known. The titration of the complex with ethidium by the other drug is monitored by fluorescence spectroscopy ${ }^{69-70}$. This experiment has been achieved with Hoechst 33258 and netropsin, to check for a further confirmation of the greater affinity of netropsin to our duplex: netropsin should then replace the ethidium molecules more readily than Hoechst 33258. The results displayed in figure 12 show that this is indeed the case. The (duplex + netropsin $+n$ ethidium) series is taking more readily the precedence over the (duplex $+\mathrm{n}$ ethidium) series than does the (duplex + Hoechst $+\mathrm{n}$ ethidium) one. Furthermore, an observed contribution of the series (duplex +2 Hoechst + n ethidium) at highest Hoechst concentrations (data not shown for clarity of the figure) is in agreement with the observation of the 2:1 complex noted above. The fact that the duplex is bound to intercalators and minor groove binders at the same time is compatible with the preference of the former for GC regions and of the latter for the ATAT site, but we cannot either rule out the possibility of non-specific aggregation of ethidium on the (duplex + minor groove binder) complex during the electrospray process. 


\section{Conclusion}

Our study of the non-covalent interaction between duplex DNA and various drugs has demonstrated the ability of mass spectrometry to distinguish between the specific and nonspecific interactions present in the solution phase. The potential uses of mass spectrometry as a rapid drug screening method has been revealed by our study of minor groove complexes. First, the stoechiometry of the complex(es) present in solution is readily assigned in a single experiment. Second, relative affinities for a particular sequence can be deduced by competition experiments with a small amount of material. If validated with a wider panel of drugs, such a protocol could be used as a support tool for drug design by the direct comparison of a modified molecule to its original form to qualitatively determine the improvement in binding affinity. 


\section{Acknowledgements}

Many thanks to professor C. Houssier (CHIMAPHY, University of Liege) for his advises about the spectroscopic measurements and their interpretation in the light of their solution phase properties. Thanks are also due to J. A. Langridge (Micromass, Manchester, UK) for his skilled demonstration on the Q-TOF with our samples, and to C. Bailly (INSERM, Lille, France) for drug supply and fruitful discussion. One author (V.G.) is grateful to the F.N.R.S. (Fonds National de la Recherche Scientifique) for her fellowship. 


\section{References}

1. M. Yamashita and J. B. Fenn, J. Phys. Chem, 88, 4451 (1984).

2. M. Whitehouse, R. N. Dreyer, M. Yamashita and J. B. Fenn, Anal. Chem, 57, 675 (1985)

3. M. Karas, D. Bachmann, U. Bahr and F. Hillenkamp, Int. J. Mass Spectrom. Ion. Proc, 78, 53 (1987).

4. M. Karas and F. Hillenkamp, Anal. Chem, 60, 2299 (1988).

5. M. Przybylski and M. O. Glocker, Angew. Chem. Int. Ed. Engl, 35, 806 (1996).

6. R. D. Smith, J. E. Bruce, Q. Wu and Q. P. Lei, Chem. Soc. Rev, 26, 191 (1997).

7. B. N. Pramanik, P. L. Bartner, U. A. Mirza, Y.-H. Liu and A. K. Ganguly, J. Mass Spectrom, 33, 911 (1998).

8. J. A. Loo and R. R. Ogorzalek Loo, in Electrospray Ionization Mass Spectrometry, ed. by R. B. Cole, pp. 385-419, Wiley, New York (1997).

9. Protein and Peptide Analysis by Mass Spectrometry, ed. by J.R. Chapman, Humana Press, Totowa (1996).

10. P. Roepstorff, Curr. Opin. Biotechnol, 8, 6 (1997).

11. D. L. Smith and Z. Zhang, Mass Spectrom. Rev, 13, 411 (1994).

12. J. A. Loo, Mass Spectrom. Rev, 16, 1 (1997).

13. P. F. Crain, in Electrospray ionization mass spectrometry, ed. by R. B. Cole, pp. 421457, Wiley, New York (1997).

14. P. F. Crain and J.A. McCloskey, Curr. Opin. Biotechnol, 9, 25 (1998).

15. P. A. Limbach, Mass Spectrom. Rev, 15, 297 (1996).

16. E. Nordhoff, F. Kirpekar and P. Roepstorff, Mass Spectrom. Rev, 15, 67 (1996).

17. J. C. Schultz, C. A. Hack and W. H. Benner, J. Am. Soc. Mass Spectrom, 9, 305 (1998).

18. B. Ganem, Y.-T. Li and J. D. Henion, Tetrahedron Lett. 34, 1445 (1993).

19. K. J. Light-Wahl, D. L. Springer, B. E. Winger, C. G. Edmonds, D. G. Camp, B. D. Thrall and R. D. Smith, J. Am. Chem. Soc, 115, 803 (1993).

20. E. Bayer, T. Bauer, K. Schmeer, K. Bleicher, M. Maier and H.-J. Gaus, Anal. Chem, 66, 3858 (1994).

21. M. J. Doktycz, S. Habibi-Goudarzi and S. A. McLuckey, Anal. Chem. 66, 3416 (1994).

22. J. Ding and R. J. Anderegg, J. Am. Soc. Mass Spectrom, 6, 159 (1995). 
23. P. D. Schnier, J. S. Klassen, E. F. Strittmatter and E. R. Williams, J. Am. Chem. Soc, 120, 9605 (1998).

24. G. Pócsfalvi, G. Di Landa, P. Ferranti, A. Ritieni,G. Randazzo and A. Marorni, Rapid Commun.Mass Spectrom, 11, 265 (1997).

25. Q. Gao, X. Cheng, R. D. Smith, C. F. Yang and I. H. Goldberg, J. Mass Spectrom. 31, 31 (1996).

26. D. C. Gale, D. R. Goodlett, K. J. Light-Wahl and R. D. Smith, J. Am. Chem. Soc, 116, 6027 (1994).

27. D. C. Gale and R. D. Smith, J. Am. Soc. Mass Spectrom. 6, 1154 (1995).

28. A. Triolo, F. M. Arcamone, A. Raffaelli and P. Salvadori, J. Mass Spectrom, 32, 1186 (1997).

29. M. J. Greig, H. Gaus, L. L. Cummins, H. Sasmor and R. H. Griffey, J. Am. Chem. Soc, 117, 10765 (1995).

30. X. Cheng, P.E. Morin, A. C. Harms, J. E. Bruce, Y. Ben-David and R. D. Smith, Anal. Biochem, 239, 35 (1996).

31. B. H. Geierstranger and D. E. Wemmer, Annu. Rev. Biomol. Struct, 24, 463 (1995).

32. J. Pindur and G. Fischer, Curr. Med. Chem, 3, 379 (1996).

33. P. B. Dervan, Science, 232, 464 (1986).

34. P. A. Cassileth and R. P. Gale, Leukemia Res, 10, 1257 (1986).

35. I. Bonnard, N. Bontemps, S. Lahmy, B. Banaigs, G. Combaut, C. Francisco, P. Colson, C. Houssier, M. J. Waring and C. Bailly, Anti-Cancer Drug Design, 10, 333 (1995).

36. The search for new anticancer drugs, ed. by M. J. Waring and B. A. J. Ponder, Kluwer Academic Publishers, Doordrecht (1992).

37. J. B. Chaires, Curr. Opin. Struct. Biol, 8, 314 (1998).

38. D. E. Thurston and A. S. Thompson, Chem. Britain, 767 (August 1990).

39. S. Neidle, Biopolymers, 44, 105 (1997).

40. M. S. Searle, Prog. NMR Spectrosc, 25, 403 (1993).

41. P. Colson, C. Bailly and C. Houssier, Biophys. Chem, 58, 125 (1996).

42. L. A. Marky, J. G. Snyder, D. P. Remeta and K. J. Breslauer, J. Biomol. Struct. Dyn, 1, 487 (1983).

43. C. E. Bostock-Smith and M. S. Searle, Nucleic. Acid. Res, 27, 1619 (1999).

44. M. L. Doyle, Curr. Opin. Biotechnol, 8, 31 (1997).

45. J. B. Chaires, Biophys. Chem, 64, 15 (1997). 
46. R. Wing, H. Drew, T. Takano, C. Broka, S. Tanaka, K. Itakura and R. E. Dickerson, Nature, 287, 755 (1980).

47. H. Drew, R. Wing, T. Takano, C. Broka, S. Tanaka, K. Itakura and R. E. Dickerson Proc. Natl. Acad. Sci. USA, 78, 2179 (1981).

48. R. E. Dickerson, M. L. Kopka and P. Pjura, Proc. Natl. Acad. Sci. USA, 80, 7099 (1983).

49. R. E. Dickerson and H. Drew, J. Mol. Biol, 149, 761 (1981).

50. R. H. Griffey, H. Sasmor and M. J. Greig, J. Am. Soc. Mass Spectrom, 8, 155 (1997).

51. R. D. Smith and K. J. Light-Wahl, Biol. Mass Spectrom, 22, 493 (1993).

52. S. A. McLuckey and D. E. Goeringer, J. Mass Spectrom, 32, 461 (1997).

53. J. G. Pelton and D. E. Wemmer, Proc. Natl. Acad. Sci. USA, 86, 5723 (1989).

54. J. G. Pelton and D. E. Wemmer, J. Am. Chem. Soc, 112, 1393 (1990).

55. K. R. Fox, Nucleic. Acid. Res, 20, 6487 (1992).

56. S. Frau, J. Bernadou and B. Meunier, Bull. Soc. Chim. Fr, 133, 1053 (1996).

57. F. G. Loontiens, P. Regenfuss, A. Zechel, L. Dumortier and R. M. Clegg, Biochemistry, 29, 9029 (1990).

58. I. Haq, J. E. Ladbury, B. Z. Chowdhry, T. C. Jenkins and J. B. Chaires, J. Mol. Biol, 271, 244 (1997).

59. J. A. Loo, D. D. Holsworth and R. S. Root-Bernstein, Biol. Mass Spectrom, 23, 6 (1994).

60. J. A. Loo, P. Hu, P. McConnell, W. T. Mueller, T. K. Sawyer and V. Thanabal, J. Am. Soc. Mass Spectrom, 8, 234 (1997).

61. Y.-L. Chen, J. M. Campbell, B. A. Collings, L. Koenermann and D. J. Douglas, Rapid Commun. Mass Spectrom, 12, 1003 (1998).

62. H.-K. Lim, Y. L. Hsieh, B. Ganem and J. D. Henion, J. Mass Spectrom, 30, 708 (1995).

63. C. V. Robinson, E. W. Chung, B. B. Kragelund, J. Knudsen, R. T. Aplin, F. M. Poulsen and C. M. Dobson, J. Am. Chem. Soc, 118, 8646 (1996).

64. T. J. D. Jorgensen, P. Roepstorff and A. J. R. Heck, Anal. Chem, 70, 4427 (1998).

65. D. M. Crothers, Biopolymers, 10, 2147 (1971).

66. M. L. Kopka, C. Yiin, D. Goodsell, P. Pjura and R. E. Dickerson, Proc. Natl. Acad. Sci. USA, 82, 1376 (1985).

67. M. Coll, J. Aymani, G. A. Van der Marel, J. H. Van Boom, A. Rich and A. H.-J. Wang, Biochemistry, 28, 310 (1989). 
68. M. A. A. F. de CT Carrondo, M. Coll, J. Aymani, A. H.-J. Wang, G. A. Van der Marel, J. H. Van Boom and A. Rich, Biochemistry, 28, 7849 (1989).

69. F. A. Tanious, J. Spychala, A. Kumar, K. Greene, D. W. Boykin and W. D. Wilson, J. Biomol. Struct. Dyn, 11, 1063 (1994).

70. M. Lee, A. L. Rhodes, M. D. Wyatt and S. Forrow, J.A. Hartley, Biochemistry, 32, 4237 (1993). 


\section{Legends to Schemes and Figures}

\section{Scheme 1}

Chemical structure of DNA minor groove binders

\section{Scheme 2}

Chemical structure of DNA intercalating agents

\section{Figure 1}

ESI spectra of duplex solutions recorded with Q-TOF at different duplex concentrations, all in aqueous $50 \mathrm{mM} \mathrm{NH}_{4} \mathrm{Ac}$ (no organic solvent added). The single strands are noted $\mathrm{C}$ and $\mathrm{G}$ and (GC) is the specific duplex. The zoom on the (GC) $)^{5-}$ species shows the distribution of sodium adducts in each case. Spectra obtained with a LCQ, using 20\% methanol in water, are identical.

\section{Figure 2}

CID spectra on (a) the duplex (GC) ${ }^{5-}$ at $25 \mathrm{~V}$ energy before entering the quadrupole and (b) the duplex $(\mathrm{GC})^{6-}$ at $30 \mathrm{~V}$. The single strands are noted $\mathrm{C}$ and $\mathrm{G}$.

\section{Figure 3}

Thermal denaturation curves of the duplex in $10 \mathrm{mM} \mathrm{NH}_{4} \mathrm{Ac}$ ( ) and in $50 \mathrm{mM} \mathrm{NH}_{4} \mathrm{Ac}($ ). The inflection points $\left(\mathrm{T}_{\mathrm{m}}\right)$ are located at $35^{\circ} \mathrm{C}$ and $48^{\circ} \mathrm{C}$ respectively. The absorbance values have been normalized to allow a visual comparison of the curves.

\section{Figure 4}

Compared full scan Q-TOF spectra of a freshly prepared $10 \mu \mathrm{M}$ duplex solution in $50 \mathrm{mM}$ $\mathrm{NH}_{4} \mathrm{Ac}$ (a) and a $10 \mu \mathrm{M}$ solution partially denatured for 12 hours at $35^{\circ} \mathrm{C}$ in $10 \mathrm{mM} \mathrm{NH} \mathrm{N}_{4} \mathrm{Ac}$ (b). The conventions are the same as in previous figures.

\section{Figure 5}

Full scan ESI-ion trap spectra of equimolar (10-10 $\mu \mathrm{M})$ duplex-drug mixtures. Drugs are (a) netropsin, (b) berenil, (c) Hoechst 33258, (d) DAPI and (e) Distamycin A. 


\section{Figure 6}

Plots of the relative intensities of the different species versus the drug molar fraction added to a $10 \mu \mathrm{M}$ duplex solution for (a) netropsin, (b) Hoechst 33258 and (c) distamycin A. The filled circles ( ) represent the intensity of the duplex (sum of the 5- and 4- charge states), the filled squares ( ) represent the intensity of the 1:1 complex (sum of the 5- and 4- charge state) and the filled triangles ( ) represent the 1:2 complex (5- charge state only, due to the limited mass range). The dots are bonded by a guideline. Spectra were recorded with the LCQ.

\section{Figure 7}

Simple ESI spectra of equimolar (10-10-10 $\mu \mathrm{M})$ mixtures of the duplex and two drugs which are thus in competition for binding to the DNA. Drug pairs are (a) DAPI/Hoechst, (b) berenil/Hoechst, (c) DAPI/netropsin and (d) Hoechst/netropsin. The latter has been recorded with the Q-TOF, while the formers with the LCQ, with a constant mass range of [1,000$2,000]$ (only a portion of the spectra is shown, for the clarity of the figure).

\section{Figure 8}

Thermal denaturation curves of the duplex ( ), its complex with Hoechst 33258 ( ) and netropsin ( ). All solutions were in $50 \mathrm{mM} \mathrm{NH} 4$ Ac. The absorbance values have been normalized to allow a visual comparison of the curves.

\section{Figure 9}

CID spectra on the (duplex + Hoechst) ${ }^{5-}$ (top) and (duplex + netropsin) $)^{5-}$ (bottom) species, both recorded at $30 \mathrm{eV}$ acceleration energy (laboratory frame).

\section{Figure 10}

Drawings illustrating the interactions between Hoechst 33258 (left) and netropsin (right) with the self-complementary d(CGCGATATCGCG) $)_{2}$ dodecamer. The hydrogen bonding interactions between the drug and the dodecamers are shown by dotted lines. (Crystallographic data reproduced from ref 68: M. A. A. F. de CT Carrondo et al., Biochemistry, 28, 7849 (1989)).

\section{Figure 11}

Full scan ESI-ion trap spectra of duplex + intercalator mixtures. The duplex concentration was kept at $10 \mu \mathrm{M}$. The nature of the intercalator and its concentration are noted in the top left 
hand corner of each spectrum. The stoechiometries of the observed species are noted $(\mathrm{GC}+\mathrm{n})$, $n$ being the number of added intercalator molecules.

\section{Figure 12}

Full scan ESI-ion trap spectra of the competition experiments between ethidium and netropsin (left) or Hoechst (right) at different minor groove binder molar fractions (noted in the top left hand corner of each spectra). The series are labeled as following: for the lone duplex, for the (duplex $+\mathrm{n}$ ethidium) series, and for the (duplex + minor groove binder $+\mathrm{n}$ ethidium) series. 
Scheme 1

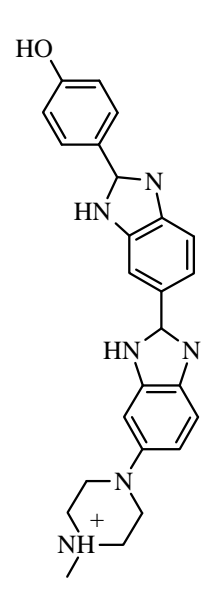

Hoechst 33258 (425)
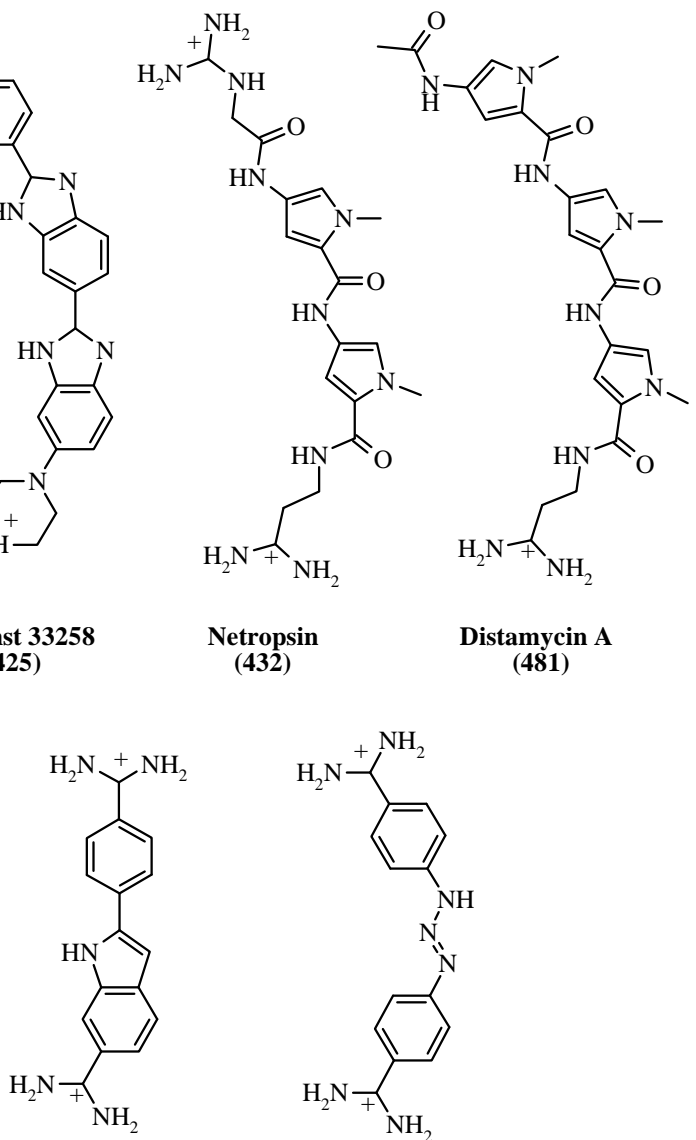

Netropsin (432)

Distamycin A (481)
DAPI
(278)
Berenil
(281)

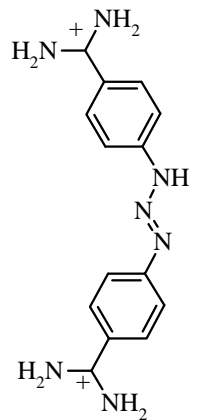


Scheme 2
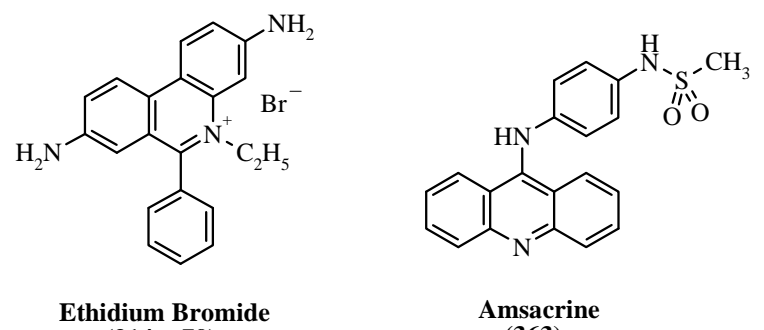

Ethidium Bromide (314 + 79)

(363)<smiles>O=C1c2cccnc2-c2nccc3c2c1nc1ccccc13</smiles>

Ascididemin

(286) 
Figure 1

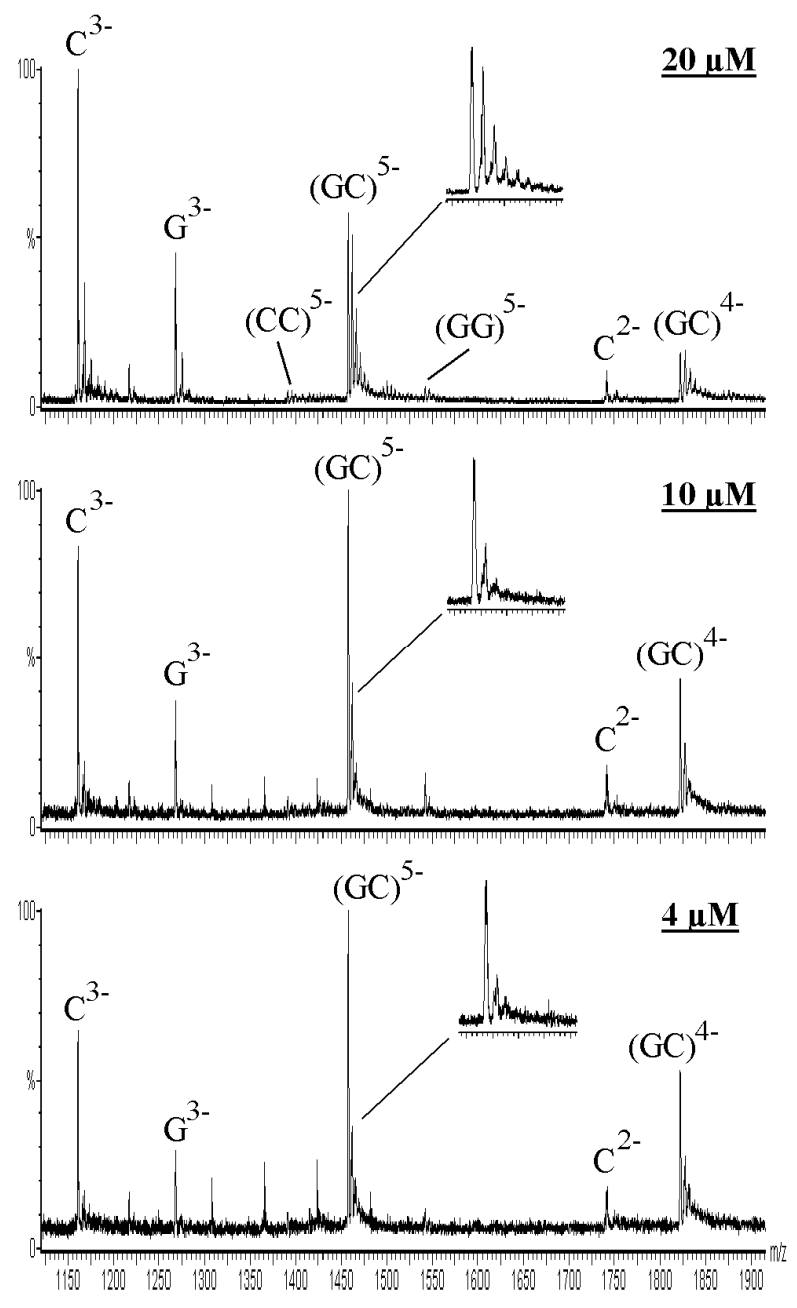


Figure 2

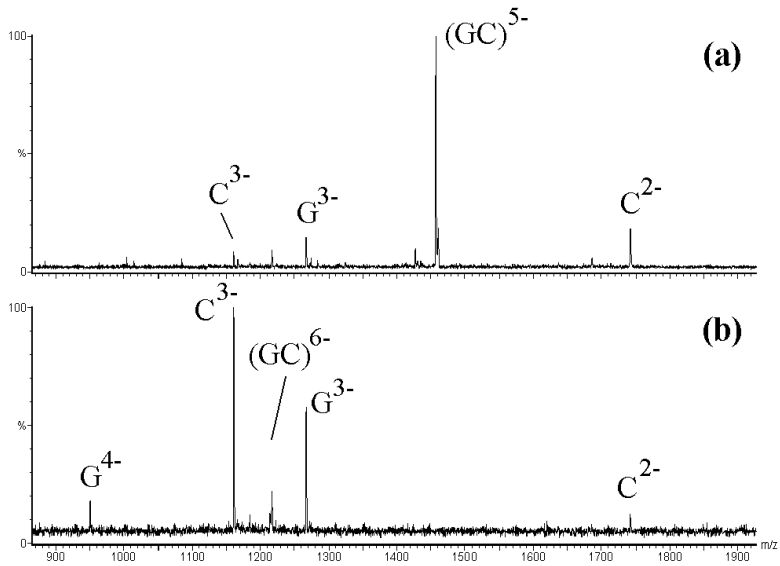


Figure 3

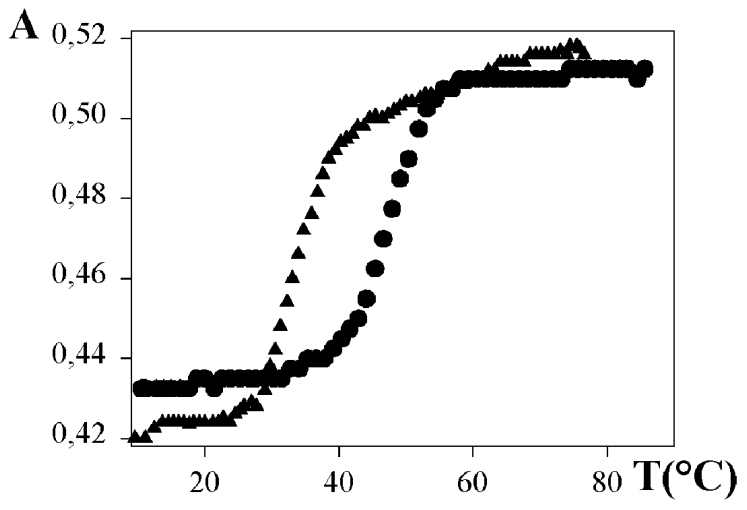


Figure 4

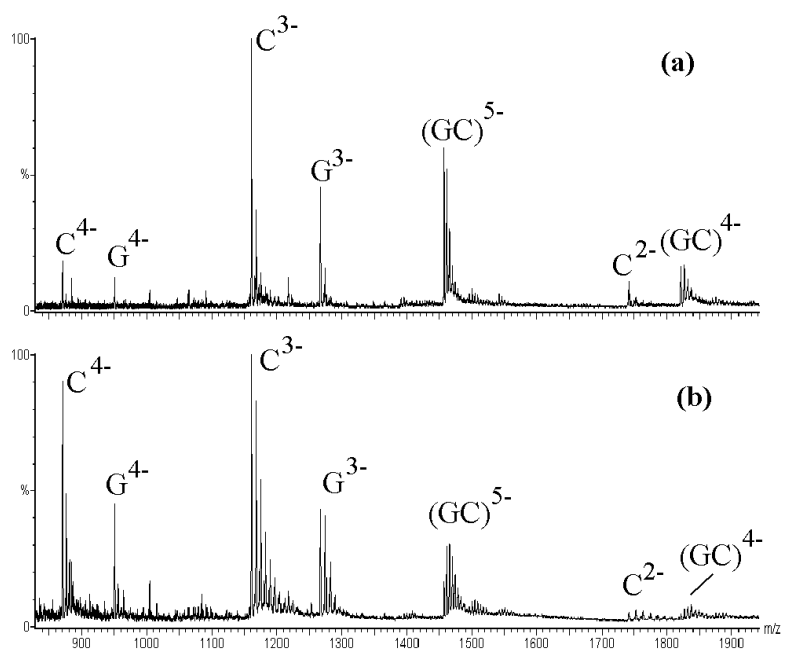


Figure 5
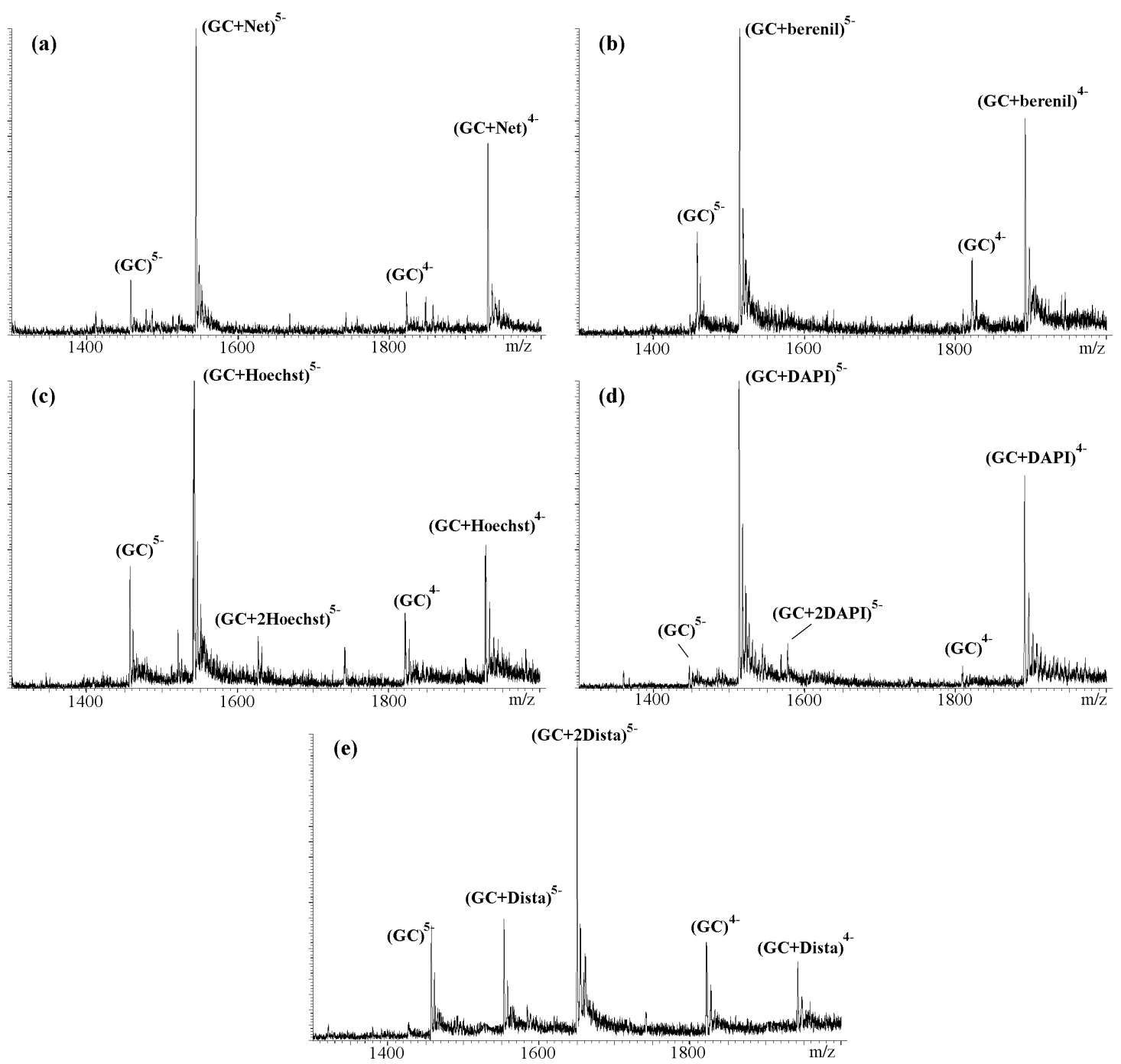
Figure 6
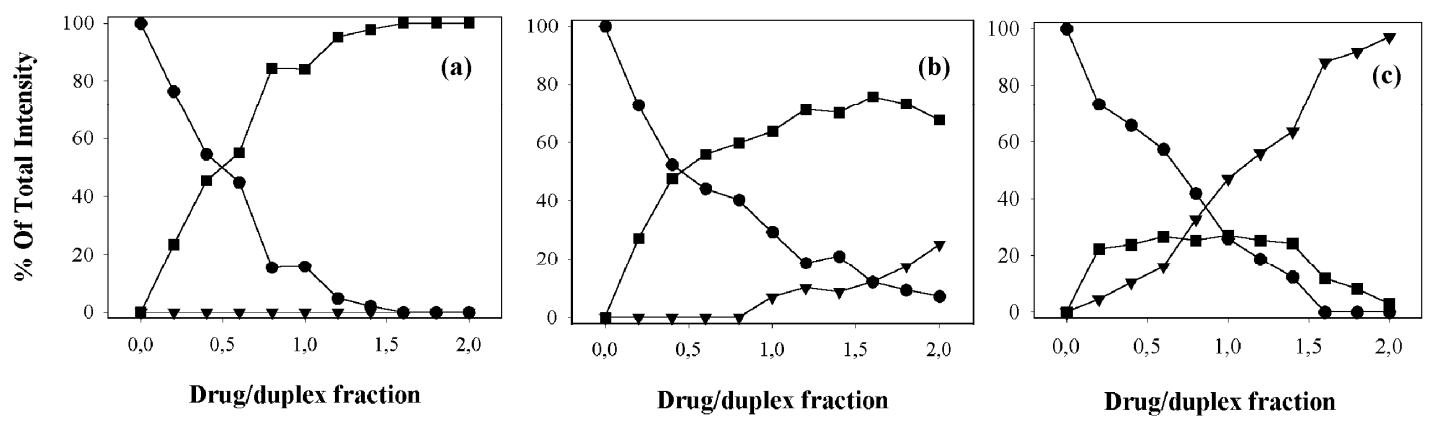
Figure 7

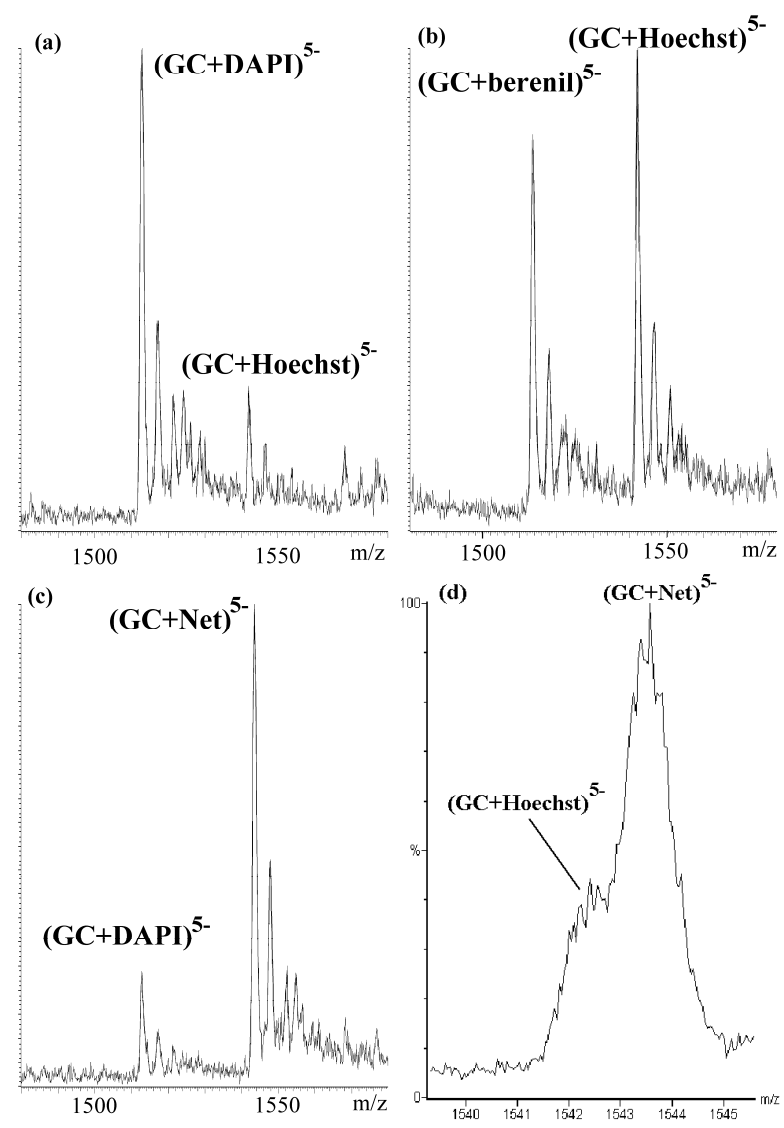


Figure 8

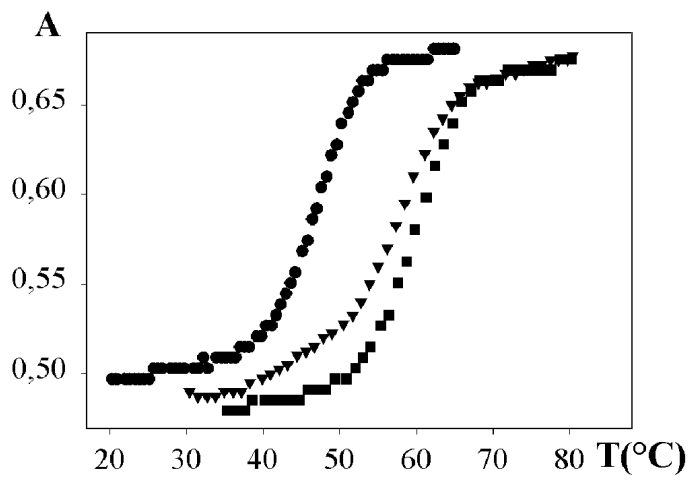


Figure 9

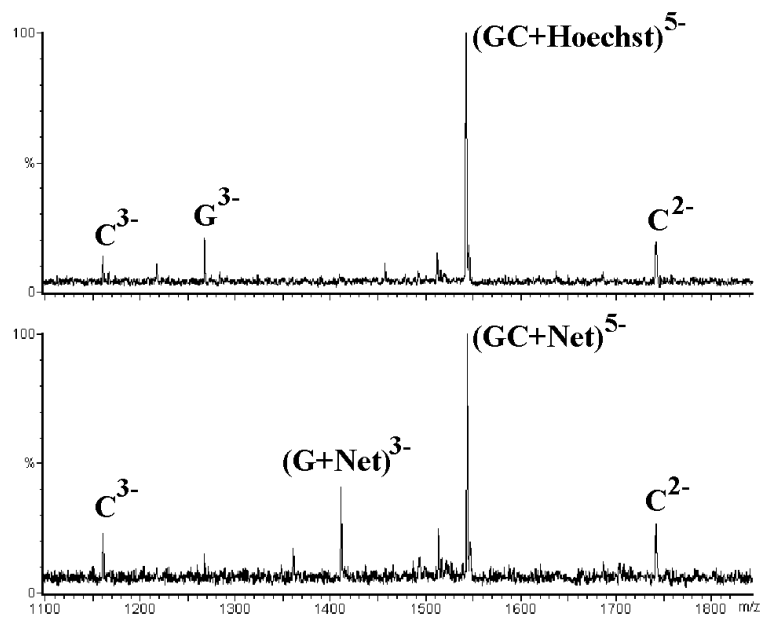


Figure 10

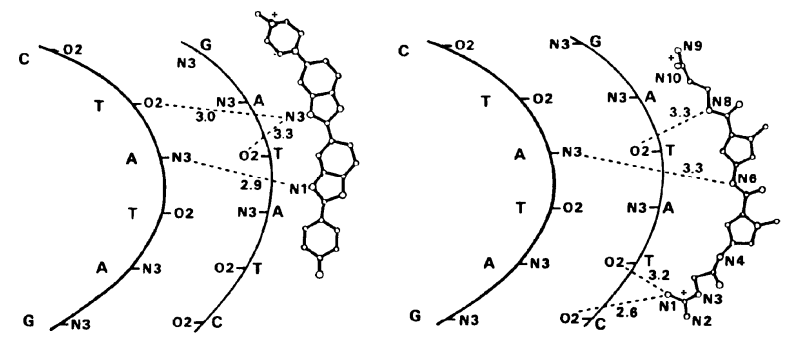


Figure 11
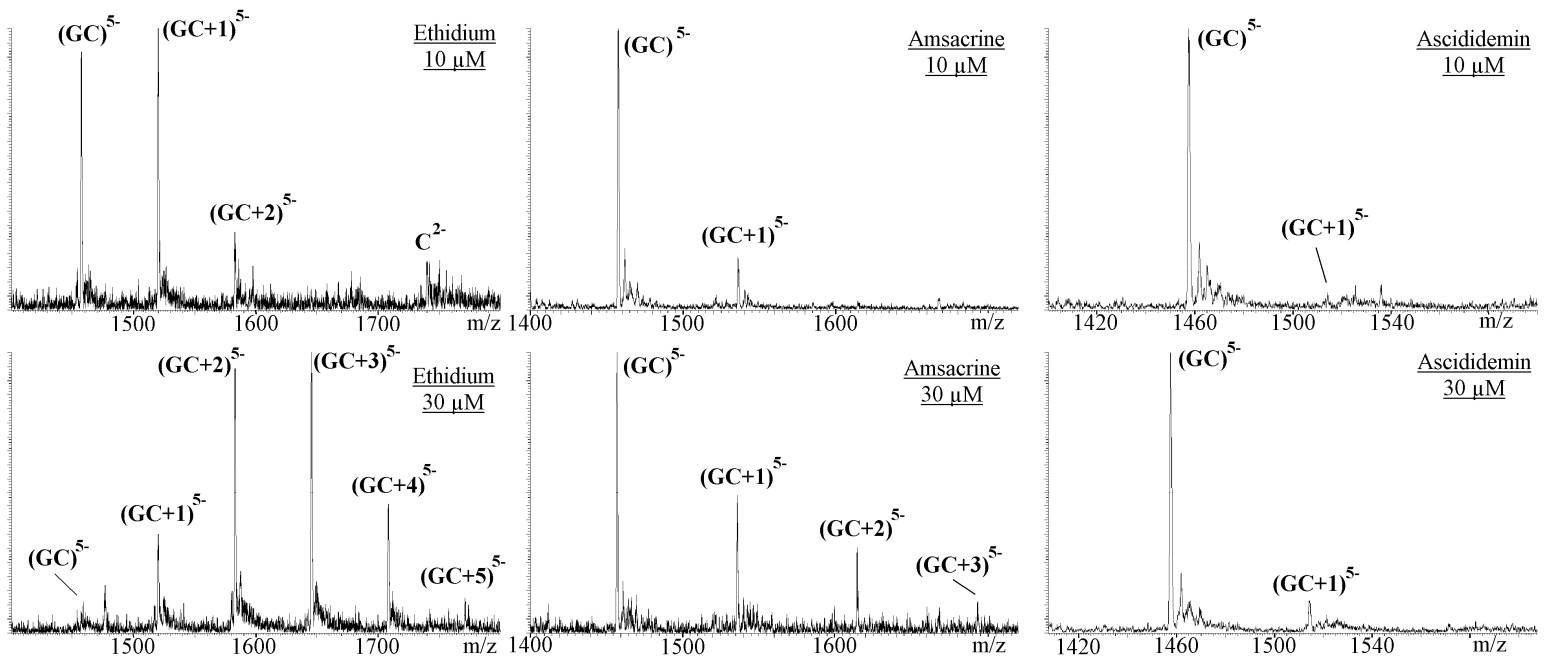
Figure 12

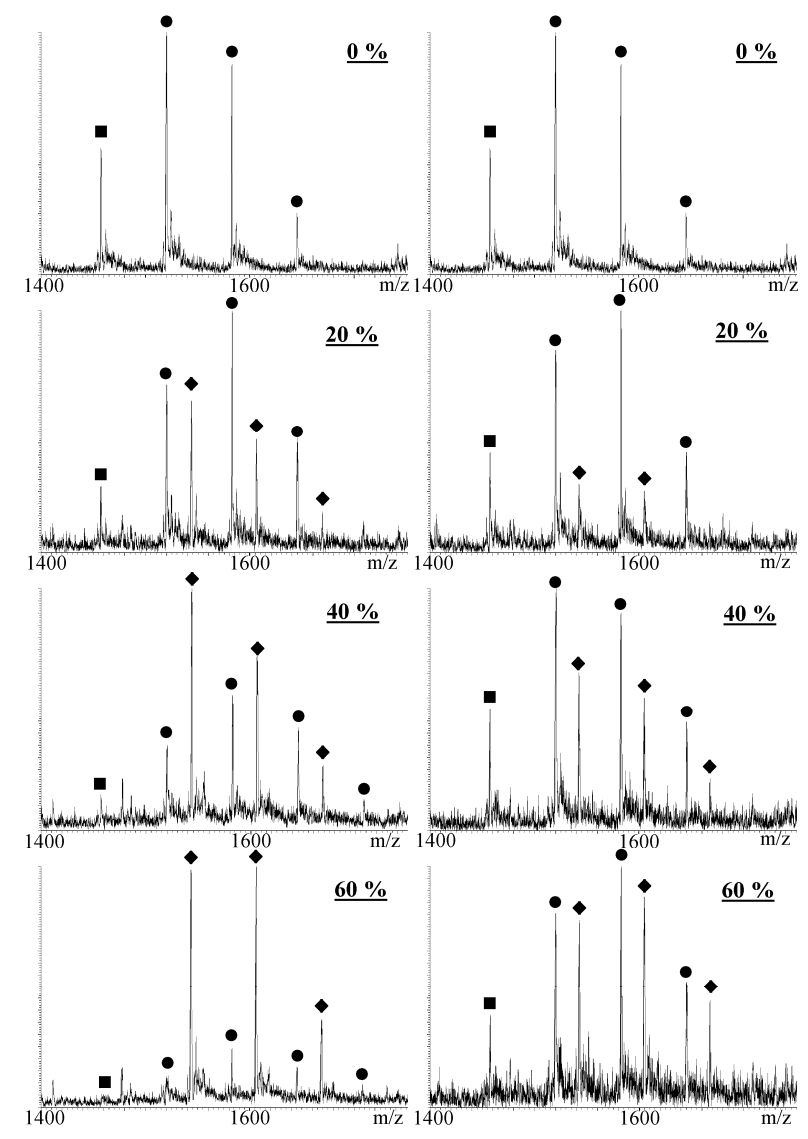

